\title{
Validez de constructo de la escala para evaluar la adherencia a procesos de neurorrehabilitación funcional (SMAN) en personas adultas
}

\author{
Construct validity of the Scale to Measure Adherence to functional \\ Neurorehabilitation processes (SMAN) in adults
}

\author{
Validade de construto da escala para avaliar a aderência a processos de \\ Neuroreabilitação Funcional (NRF) em adultos
}

\begin{abstract}
Carlos Andrés Quiroz-Mora', Mauricio Hernández-Carrillo², Ayda Zulamy Agudelo-Martínez³, Carlos A. Medina-Riaño4
1 Fisioterapeuta, Msc en Neurorrehabilitación, Msc en Epidemiología. Institución Universitaria Escuela Nacional del Deporte. Cali, Colombia. enzoandrew@hotmail.com. ORCID: https://orcid.org/0000-0003-4106-3019

2 Estadístico, Msc en Epidemiología. Institución Universitaria Escuela Nacional del Deporte. Cali, Colombia. mauriciohc@gmail.com. ORCID: https://orcid.org/0000-0001-8816-7726

3 Fisioterapeuta, Msc en Neurorrehabilitación. Institución Universitaria Escuela Nacional del Deporte. Cali, Colombia. zulamy.agudelo@ endeporte.edu.co. ORCID: https://orcid.org/0000-0001-6771-0240

$4 \quad$ Fisioterapeuta, Msc en Neurorrehabilitación. Institución Universitaria Escuela Nacional del Deporte. Cali, Colombia. carlos.medina@ endeporte.edu.co ORCID: https://orcid.org/0000-0003-3840-8151
\end{abstract}

Recibido: 13/08/2020. Aprobado: 15/07/2021. Publicado: 13/08/2021

Quiroz-Mora CA, Hernández-Carrillo M,Agudelo-Martínez AZ, Medina-Riaño CA. Validez de constructo de la escala para evaluar la adherencia a procesos de neurorrehabilitación funcional (SMAN) en personas adultas. Rev. Fac. Nac. Salud Pública. 2021;39(3):e341716. DoI: https://doi.org/10.17533/udea.rfnsp.e341716

\section{Resumen}

Objetivo: Determinar la validez de constructo de la escala para evaluar la adherencia a procesos de neurorrehabilitación funcional (SMAN) en personas adultas. Metodología: Estudio instrumental, con un diseño transversal, en el que se hace uso del método de análisis factorial confirmatorio para un modelo inicial de 5 factores y 20 ítems. Se utilizó el método de máxima verosimilitud y se realizaron pruebas de normalidad univariada y multivariada. El modelo hipotetizado se valoró con medidas de ajuste absoluto, ajuste incremental y ajuste de la parsimonia. Para el modelo definitivo, se calcularon estadísticos de fiabilidad, y validez convergente y discriminante, a través de matriz de correlaciones. Resultados: Para el modelo inicial (M0), los índices de modificación mostraron cargas cruzadas, pertenecientes a los constructos de "discapacidad" y "paciente"; por tanto, se hizo una revisión teórica de los ítems del instrumento y se eliminaron dos ítems redundantes, uno en cada factor. Posterior a la reespecificación del modelo (M2), la SMAN quedo conformada por 5 factores y 18 ítems. Conclusión: la SMAN posee propiedades psicométricas destacables y confiables para la medición de la adherencia en poblaciones con déficit funcional asociado a condición neurológica.

---------Palabras clave: cumplimiento y adherencia al tratamiento, encuestas y cuestionarios, estudios de validación, rehabilitación neurológica. 


\begin{abstract}
Objective: To determine the construct validity of the Scale to Measure Adherence to functional Neurorehabilitation processes (SMAN) in adults. Methodlogy: Instrumental study, with a cross-sectional design, in which the confirmatory factor analysis method was used for an initial model of 5 factors and 20 items. The maximum likelihood method was used and univariate, and multivariate normality tests were performed. The hypothesized model was evaluated with absolute, incremental and parsimony adjustment measures. For the definitive model, reliability and convergent and discriminant validity were calculated through a correlation matrix. Results: For the initial model (M0), the modification indices showed
\end{abstract}

cross-loading related to constructs of "disability" and "patient". Therefore, a theoretical review of instrument items was made, and two redundant items were eliminated, one in each factor. After model re-specification (M2), SMAN was constituted by 5 factors and 18 items. Conclusion: SMAN has remarkable and reliable psychometric properties to measure adherence in populations with functional deficits associated with neurological conditions.

-------Keywords: compliance and adherence to treatment, surveys and questionnaires, validation studies, neurological rehabilitation.

\section{Resumo}

Objetivo: Determinar a validade de construto da escala para avaliar a aderência a processos de neuroreabilitação funcional (NRF) em adultos. Metodologia: Estudo instrumental com um desenho transversal, no qual se utiliza o método de análise fatorial confirmatória para um modelo inicial de 5 fatores e 20 itens. Foi utilizado o método de máxima verossimilhança e foram realizados testes de normalidade univariado e multivariado. O modelo hipotetizado foi avaliado com medidas de ajuste absoluto, ajuste incremental e ajuste da parcimônia. Para o modelo definitivo, foram calculados dados estatísticos de confiabilidade e validade convergente e discriminante, através de matriz de correlações. Resultados: Para o modelo inicial (MO), os índices de modificação mostraram cargas cruzadas pertencentes aos construtos de "incapacidade" e "paciente"; portanto foi feita uma revisão teórica dos itens do instrumento e foram eliminados dois itens redundantes, um em cada fator. Posterior à reespecificação do modelo (M2), a NRF foi formada por 5 fatores e 18 itens. Conclusão: ARNF possui propriedades psicométricas destacáveis e confiáveis para a medição da aderência em populações com déficit funcional associado à condição neurológica.

-Palavras-chave: Cumprimento e aderência ao tratamento, Pesquisas e questionários, Estudos de validação, Reabilitação neurológica.

\section{Introducción}

Las personas con deficiencias del sistema nervioso central o del periférico sufren con frecuencia de impedimentos neurológicos, déficit funcional y discapacidad, que llevan a la necesidad de elaborar esquemas de rehabilitación para la recuperación de sus patrones básicos de movimiento. Sin embargo, en la mayoría de los países en vía de desarrollo, los procesos de rehabilitación y seguimiento son con frecuencia deficientes. Esta situación podría estar relacionada con diferentes actores, como el sistema de salud, las familias y los usuarios, con respecto a las estrategias para favorecer la adherencia a los procesos terapéuticos y los altos costos que implica la no continuidad de los usuarios en dichos procesos. Por tanto, la comprensión de este fenómeno permitiría integrar acciones que maximicen la dinámica de los procesos de atención y mejorar la prestación de los servicios de salud para el individuo y sus redes de apoyo [1].
La adherencia a los procesos terapéuticos es un fenómeno complejo, que requiere la comprensión desde diferentes perspectivas: evaluativas, tecnológicas, económicas, sociales y culturales [2]. En efecto, la Organización Mundial de la Salud ha planteado cinco dimensiones que afectan la adherencia a procesos de tratamiento a largo plazo: sociodemográficos, factores del sistema o el equipo de asistencia sanitaria, relacionados con el tratamiento, relacionados con la enfermedad y factores relacionados con el paciente [3]. Estas dimensiones son también consideradas en aquellos procesos necesarios de interacción para la rehabilitación funcional, y que a largo plazo dependen de la capacidad residual generada por los déficits neurológicos sucedidos en el individuo [2,4-6].

La evidencia empírica y la reflexión teórica han demostrado la relación existente entre la adherencia 
terapéutica y los procesos de neurorrehabilitación funcional, en articulación con las dimensiones enunciadas. En factores sociodemográficos, aspectos como los recursos económicos [6,7], el círculo social $[8,9]$, el estigma social [10] y el apoyo de la familia $[9,11]$ han mostrado un vínculo funcional para la adherencia terapéutica. Para los factores relacionados con el sistema o el equipo de asistencia sanitaria, se ha demostrado la influencia de los servicios de salud [12], la idoneidad profesional [13-15], el apoyo dado por los profesionales de la salud $[6,16]$ y el interés manifiesto por el personal asistencial [12,15] en el cumplimiento y la adherencia de los usuarios. Asimismo, factores como el proceso de neurorrehabilitación funcional, la frecuencia de las terapias $[17,18]$, la percepción del cumplimiento por parte del profesional de la salud $[5,6]$, la percepción de mejoría por parte del usuario [19-21] y las complicaciones o los efectos secundarios resultado de los procesos terapéuticos [18,22,23] muestran un impacto directo sobre el cumplimiento y la adherencia a dichos procesos. Igualmente, en el factor que tiene que ver con la discapacidad, se han descrito relaciones de la adherencia terapéutica con la condición funcional [24], la progresión de la enfermedad [22], la severidad de la enfermedad $[15,22,25]$ y las comorbilidades $[22,26,27]$. Por último, respecto a los factores asociados al paciente, el nivel de motivación en el proceso de rehabilitación [12,21], la importancia dada en el proceso de recuperación [11,28], la comprensión del proceso $[21,29]$ y la esperanza $[15,30]$ son considerados elementos importantes para la adherencia a procesos de neurorrehabilitación funcional.

La medición de la adherencia constituye un elemento importante para la toma de decisiones en los servicios de salud, lo que posibilita dinamizar el proceso de rehabilitación, al permitir comprender el valor aportado que imprimen los factores sociales, culturales, económicos, políticos y del sistema de salud a la adherencia desde una perspectiva más global [31-34].

En el ámbito de la rehabilitación, es escaza la evidencia de instrumentos, cuestionarios o escalas creadas para determinar la adherencia a procesos terapéuticos [35]. Dado lo anterior, se cuenta con la escala para la medición de la adherencia a procesos de neurorrehabilitación (Scale to Measure Adherence to the Neurorehabilitation, SMAN), desarrollada entre el 2012 y el 2020. Inicialmente, se efectuó un proceso de validez interna de la escala SMAN, con la creación de los constructos y las preguntas, medido con el estadístico Alfa de Cronbach (AC) [36]; en segunda instancia, se realizó un proceso de confiabilidad inter e intraevaluador, medido a través del índice kappa de Cohen [37]. Finalmente, se planteó la necesidad de determinar la validez del constructo de la SMAN, por medio de un análisis factorial confirmatorio (AFC), dada la falta, en el ámbito de rehabilitación, de una escala con propiedades psicométricas destacables y confiables para la medición de la adherencia a procesos terapéuticos en poblaciones con déficit funcional asociado a condición neurológica.

\section{Metodología}

Estudio instrumental, con un diseño transversal, en el que se hace uso del método de AFC para un modelo inicial de 5 factores y 20 ítems.

\section{Participantes}

La población estuvo constituida por personas que asistieron a procesos de neurorrehabilitación en tres centros de rehabilitación privados y uno público, ubicados en la ciudad de Cali.

En relación con las características de los participantes del estudio, el $60 \%$ eran hombres y la edad media de 44 años (desviación estándar: 18,7) para ambos sexos. El $45 \%$ de las personas eran solteras, el $35 \%$ pertenecía al nivel socioeconómico medio-bajo (estrato 3 en Colombia), el $58 \%$ hacía parte del régimen contributivo (pago de cotización para prestación de servicios en salud), el $20 \%$ eran trabajadores independientes, el $38 \%$ contaba con estudios universitarios, y la condición clínica más frecuente fue la enfermedad cerebrovascular y el trauma raquimedular, con una representación entre un 25 y $35 \%$, respectivamente.

\section{Muestra}

La muestra de esta investigación estuvo constituida por 100 personas, seleccionadas de forma no probabilística, que hacían parte de los servicios de neurorrehabilitación funcional de los centros de rehabilitación incluidos en el estudio.

El tamaño de la muestra para este estudio se obtuvo teniendo en cuenta los criterios descritos por Lloret et al. [38].

\section{Instrumento}

La SMAN de Quiroz [36] está integrada por 5 constructos, cada uno con 4 ítems, determinados como preguntas en escala de Likert en un rango de 1 (Nunca) a 5 (Siempre) (véase Anexo).

La adherencia se puntúa de 20 a 100 y el valor descrito se puede ubicar en rangos de adherencia como: nula (0-4 \%), baja (5-25\%), mediana (26-50\%), satisfactoria (51-94\%), alta (95-100\%).

La fiabilidad interna de la escala reportada por Quiroz [36] tuvo un valor de AC de 0,83. La confiabilidad inter e intraevaluador tuvo un valor de índice kappa de 0,76 y 0,71; así mismo, el coeficiente de correlación de Spearman fue de 0,82 y 0,80, reportada por Quiroz y Pérez [37]. 


\section{Procedimiento}

Entre marzo y abril del año 2019 se invitó a participar en la investigación a las personas que asistieron a procesos de neurorrehabilitación funcional en cuatro centros especializados de Cali. El criterio de inclusión de los participantes en el estudio se determinó por: vinculación a los procesos de rehabilitación y aceptación de participación en la investigación a través de consentimiento informado.

Los centros especializados seleccionados para la inclusión de los participantes son los que tienen mayor afluencia de personas a procesos de neurorrehabilitación en la ciudad de Cali. La convocatoria para participar en el estudio se realizó directamente en los servicios de rehabilitación y la participación fue completamente voluntaria.

El instrumento SMAN fue suministrado a cada participante y diligenciado de forma privada; cuando el participante requirió orientación, un encuestador entrenado aclaró las dudas.

Los principios éticos que se siguieron en el presente estudio fueron: beneficencia, respeto a la autonomía, no maleficencia, justicia, fidelidad y veracidad. El aval ético de la presente investigación quedó registrado en el Acta 11 del 22 de noviembre de 2018 del Grupo de Investigación de Estudios de Salud y Sociedad (GIESS), de la Institución Universitaria Escuela Nacional del Deporte.

Como se indicó, para verificar la validez de constructo de la SMAN, se llevó a cabo un estudio instrumental, con un diseño transversal, en el que se hacía uso del método de AFC, el cual permite contrastar la validez de los constructos de los cinco factores definidos por la SMAN mediante el ajuste de un modelo que la asuma y diagnosticando su bondad de ajuste (validación de constructo) [39]. Para reducir el sesgo de información o de medición, se realizó previamente capacitación de encuestadores, quienes hicieron la aplicación del instrumento.

\section{Análisis de datos}

Para el análisis, se usó el paquete estadístico IBM $\AA$ SPSS $®$ Statistics Versión 24 e IBM ${ }^{\circledR}$ SPSS ${ }^{\circledR} \operatorname{amos}{ }^{\circledR}$ Versión 24. El modelo planteado se evaluó a través del método de máxima verosimilitud; asimismo, para determinar la normalidad univariada, se examinaron los índices de asimetría y curtosis [40], y para normalidad multivariada, se examinó el índice de Mardia [41].

Se tuvieron en cuenta los índices de modificación (Modification Index, MI) y el parámetro de cambio esperado (Expected Parameter Change, EPC) para las covarianzas, las varianzas y los pesos de regresión con valor de referencia mayor o igual que 10,00, dado que un valor inferior no daría ningún resultado significativo en el ajuste general del modelo. La modificación del modelo después del análisis inicial solo se ejecutó cuando esta cumplía con los criterios estadísticos y se ajustaba a la comprensión teórica de la SMAN $[42,43]$.

Se valoró el ajuste del modelo teniendo en cuenta las medidas de ajuste absoluto, las de ajuste incremental y las de ajuste de la parsimonia. Las medidas de ajuste absoluto fueron: chi cuadrado relativo $\left(\chi^{2} / g l\right)$, índice de bondad de ajuste (Goodness of Fit Index, GFI), índice corregido de bondad de ajuste (Adjusted Goodness of Fit Index, AGFI), error cuadrático medio estandarizado (Standardized Root Mean Square Residual, SRMR) y error de aproximación cuadrático medio (Root Mean Square Error of Approximation, RMSEA). Las medidas de ajuste incremental fueron: índice normado de ajuste (Normed Fit Index, NFI), índice de ajuste comparativo (Comparative Fit Index, CFI), índice no normalizado de ajuste o de Tucker-Lewis (Tucker-Lewis Index, TLI) y el índice de ajuste incremental (Incremental Fit Index, IFI). Las medidas de ajuste a la parsimonia fueron: índice de bondad de ajuste de parsimonia (Parsimony Goodness of Fit Index, PGFI), índice de ajuste normado de parsimonia (Parsimony Normed of Fit Index, PNFI), el criterio de información de Akaike (Akaike Information Criterion, AIC) y el índice de validación cruzada esperada (Expected Cross Validation Index, ECVI).

Los valores de referencia considerados para el ajuste del modelo fueron: $\chi^{2} / g l: \leq 2$, GFI y AGFI $=1,0$ (ajuste perfecto), SRMR $\leq 0,05$, RMSEA $\leq 0,05$, NFI y CFI $\geq 0,90$, TLI $\geq 0,95$ e IFI $\geq 0,90$, según Byrne [42] y Wheaton et al. [44]; y para las medidas de ajuste a la parsimonia, PGFI y PNFI $\geq 0,50$, y AIC y ECVI con valores inferiores después de cada reespecificación del modelo o cercanos a cero, según Byrne [42] y Hooper et al. [45].

Posterior a la reespecificación del modelo factorial planteado, se realizaron estadísticos de fiabilidad y validez. Para la fiabilidad, se midió el Ac; para la validez convergente, se usó la varianza extraída media (AVE), y para la validez discriminante, se calculó la matriz de correlaciones ( $r$ de Pearson) entre los ítems de la escala o fiabilidad en diagonal, según Hu y Bentler [46], y Henseler, Ringle y Sarstedt [47]. El valor de referencia del AC fue mayor o igual que 0,70, según Oviedo y Campo [48]. Debido a que la SMAN tiene más de un constructo y el AC no tiene en cuenta la influencia que los otros constructos pueden tener sobre el medido, como lo plantea Dunn et al. [49], se usó el índice de fiabilidad compuesta (IFC) según Fornell y Larcker [50], que tuvo en cuenta las interrelaciones de los constructos extraídos. El valor de referencia del IFC fue de mayor o igual que 0,70 , basado en Prieto y Delgado [51]. El valor de referencia para AVE fue de mayor o igual que 0,50, según Fornell y Larcker [50]. 


\section{Resultados}

El AFC propuesto estableció la relación entre cinco constructos de la SMAN, expresados en forma de variables latentes o factores: socioeconómico, sistema, rehabilitación, discapacidad y paciente, representados cada uno con cuatro variables observables o ítems (véase Figura 1).

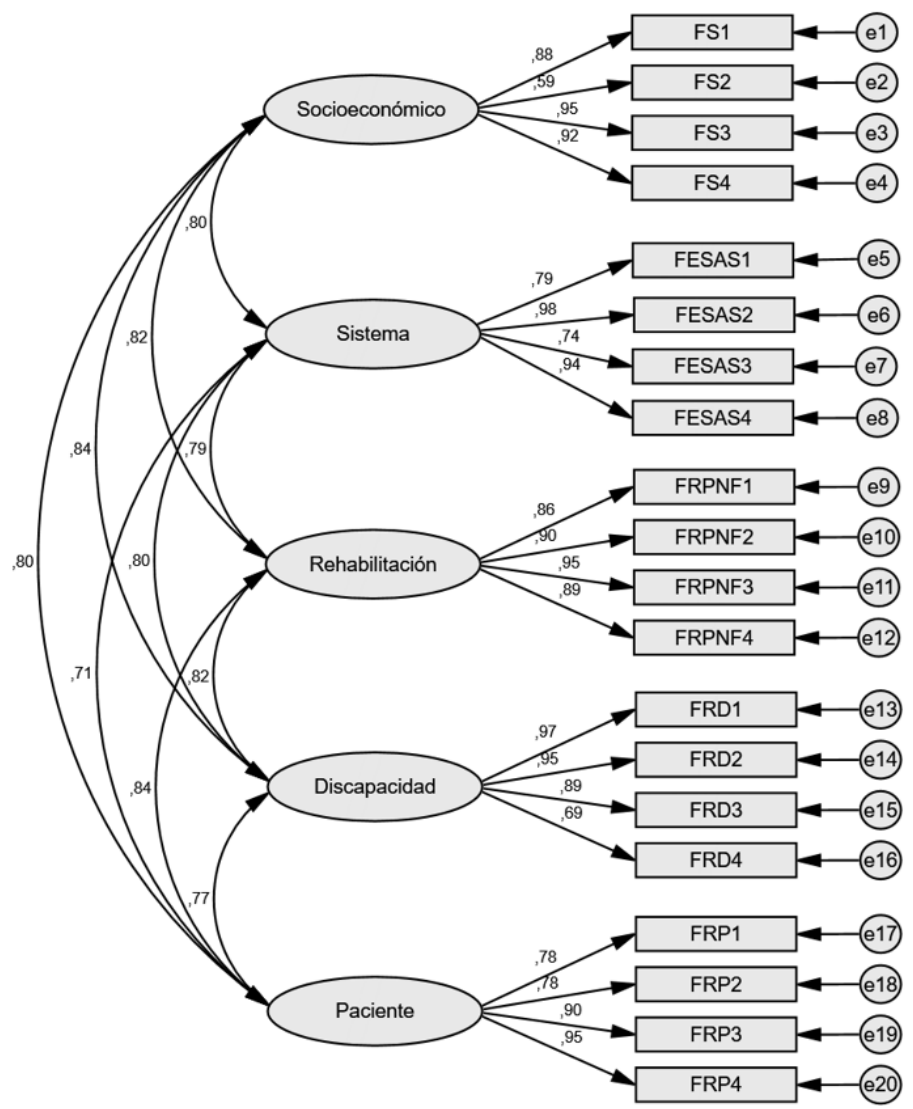

Figura 1. AFC en modelo de 5 factores y 20 ítems (M0). Se presentan las variables observadas con estimados de carga estandarizados. FS: factores socioeconómicos; FESAS: factores de equipo y sistema de asistencia sanitaria; FRPNF: factores relacionados con el proceso de neurorrehabilitación funcional; FRD: factores relacionados con la discapacidad; FRP: factores relacionados con el paciente.

Los parámetros establecidos para el AFC fueron estimados mediante el método de máxima verosimilitud. Los valores obtenidos en el índice de asimetría estuvieron entre 0,61 y 1,99 , por debajo de 2 , y para curtosis, entre 0,03 y 2,72, por debajo de 5 , según lo establecido por Ryu [40]. La normalidad multivariada, medida a través del índice de Mardia, obtuvo un valor de 20,37, inferior a 50, según lo establecido por Rodríguez y Ruiz [41]; por tanto, existe normalidad univariada y multivariada de los datos.

Para los estimados de carga estandarizados de las variables observadas, todas tuvieron valores mayores que $0,50, p<0,001$. Para el primer modelo hipotetizado (M0), se encontraron dos MI con valor mayor o igual que 10,00. Este resultado mostraba una posible cova- rianza entre las varianzas de error relacionadas con las variables indicadoras, representadas por el símbolo $(\leftrightarrow)$ : FRD4 y FRP1 (e16 ↔ e17), FRPNF3 y FRP4 (e11 $\leftrightarrow$ e20). Para FRD4 y FRP1, se muestra que el MI es 11,24 y el EPC es 0,21. Para FRPNF3 y FRP4, se muestra que el MI es 11,16 y el EPC es 0,08. En términos generales, el valor de MI sugiere que si este parámetro se estimara libremente en un análisis posterior, el valor $X^{2}$ general disminuiría en aproximadamente 11,24 y la covarianza de error estimada tomaría un valor aproximado de 0,21. Dado que las rutas de los MI mostraron cargas cruzadas, es decir, pertenecen a diferentes constructos, no se procedió a establecer rutas de regresión y se procedió a hacer una revisión de los ítems del instrumento. 
Los ítems FRD4 y FRP4 son redundantes dentro del instrumento, en los constructos de discapacidad y paciente; por tanto, se procedió a eliminarlos, especificando dos modelos (M1 y M2) para cada ítem eliminado.

Las modificaciones se realizaron en pasos sucesivos (véase Tabla 1), concluyendo en un modelo M2, compuesto por 5 constructos y 18 ítems (véase Figura 2).
Para el modelo M2, no existió diferencia entre la matriz de datos observada y el modelo hipotetizado $(p=0,12, \geq$ $0,05)$; asimismo, el modelo M2 explica las correlaciones dentro de un error promedio de 0,04 (SRMR $\leq 0,05$ ). Todos los índices de ajuste absoluto, ajuste incremental y ajuste a la parsimonia para el modelo M2 estuvieron por encima de los criterios establecidos.

Tabla 1. Especificación del modelo M0 y estadísticos de bondad de ajuste

\begin{tabular}{lcccccccccccc}
\hline \multicolumn{1}{c}{ Modelo } & $\chi^{2} /$ gl & GFI & AGFI & RMSEA & NFI & CFI & TLI & IFI & PGFI & PNFI & AIC & ECVI \\
\hline M0 original & 1,30 & 0,84 & 0,79 & 0,05 & 0,91 & 0,97 & 0,97 & 0,97 & 0,64 & 0,76 & 309,0 & 3,12 \\
M1 = M0 - FRD4 & 1,21 & 0,86 & 0,81 & 0,04 & 0,92 & 0,98 & 0,98 & 0,98 & 0,64 & 0,76 & 268,9 & 2,71 \\
M2 = M1 - FRP4 & 1,14 & 0,87 & 0,82 & 0,03 & 0,93 & 0,99 & 0,98 & 0,99 & 0,63 & 0,76 & 235,1 & 2,37 \\
Criterio de referencia & $\leq 2$ & Cerca de 1,0 & $\leq 0,05$ & $\geq 0,90$ & $\geq 0,90$ & $\geq 0,95$ & $\geq 0,90$ & $\geq 0,50$ & $\geq 0,50$ & Reducción \\
\hline
\end{tabular}

Nota: $\chi^{2} / g l$ : chi cuadrado relativo; GFI: índice de bondad de ajuste; AGFI: índice corregido de bondad de ajuste; SRMR: error cuadrático medio estandarizado; RMSEA: error de aproximación cuadrático medio; NFI: índice normado de ajuste; CFI: índice de ajuste comparativo; TLI: índice de TuckerLewis; IFI: índice de ajuste incremental; PGFI: índice de bondad de ajuste de parsimonia; PNFI: índice de ajuste normado de parsimonia; AIC: criterio de información de Akaike; Ecvi: índice de validación cruzada esperada.

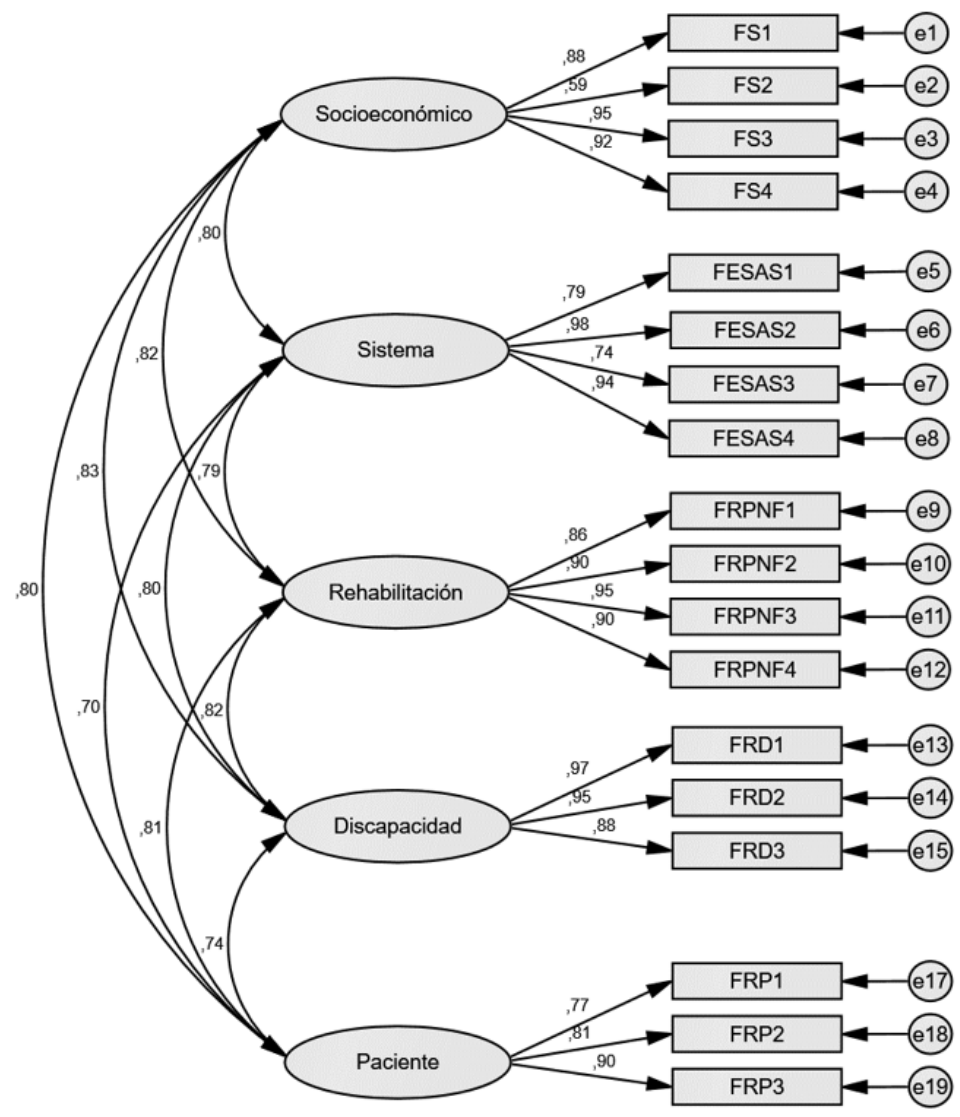

Figura 2. Modelo resultante del AFC con 5 factores y 18 ítems (M2). Se presentan las variables observadas con estimados de carga estandarizados. FS: factores socioeconómicos; FESAS: factores de equipo y sistema de asistencia sanitaria; FRPNF: factores relacionados con el proceso de neurorrehabilitación funcional; FRD: factores relacionados con la discapacidad; FRP: factores relacionados con el paciente. 
El instrumento SMAN, compuesto con 18 ítems, tuvo un valor de AC de 0,96. Los valores de AC, IFC y AVE para cada constructo estuvieron por encima del criterio de referencia (véase Tabla 2).

La validez discriminante se calculó por medio de la matriz de correlaciones entre los ítems de la escala. Existió correlación significativa, positiva y alta entre todos los constructos que componen la SMAN $(p<0,001)$.
Para Fornell y Larcker [50], un modelo posee validez discriminante si la raíz cuadrada de la AVE de cada constructo o valor sobre la diagonal es mayor que las correlaciones con el resto de constructos, lo que implica entonces que cada constructo es diferente a otro. Todos los indicadores se ajustan de forma aceptable de acuerdo con los principios establecidos, garantizando la validez discriminante de los constructos (véase Tabla 3 ).

Tabla 2. Estadísticos de fiabilidad y validez convergente de la SMAN

\begin{tabular}{lccc}
\hline \multicolumn{1}{c}{ Constructos } & AC & IFC & AVE \\
\hline Criterio de referencia & $\geq 0,70$ & $\geq 0,70$ & $\geq 0,50$ \\
FS: Factores socioeconómicos & 0,89 & 0,90 & 0,71 \\
FESAS: Factores del equipo o el sistema de asistencia sanitaria & 0,91 & 0,92 & 0,75 \\
FRPNF: Factores relacionados con el proceso de & 0,94 & 0,94 & 0,81 \\
neurorrehabilitación funcional & & & \\
FRD: Factores relacionados con la discapacidad & 0,95 & 0,95 & 0,87 \\
FRP: Factores personales & 0,87 & 0,87 & 0,68 \\
\hline
\end{tabular}

Nota: AC: Alfa de Cronbach, IFC: índice de fiabilidad compuesta, AVE: varianza extraída media.

Tabla 3. Correlación entre factores resultantes y validez discriminante de la SMAN.

\begin{tabular}{lccccc}
\hline & Socioeconómico & Sistema & Rehabilitación & Discapacidad & Paciente \\
\hline Socioeconómico & $(0,84)$ & & & & \\
Sistema & $0,79^{* * *}$ & $(0,86)$ & & & \\
Rehabilitación & $0,82^{* * *}$ & $0,78^{* * *}$ & $(0,90)$ & & \\
Discapacidad & $0,83^{* * *}$ & $0,79^{* * *}$ & $0,82^{* * *}$ & $(0,93)$ & \\
Paciente & $0,80^{* * *}$ & $0,70^{* * *}$ & $0,81^{* * *}$ & $0,73^{* * *}$ & $(0,82)$ \\
\hline
\end{tabular}

Correlaciones significativas ${ }^{* * *} p<0,001$

\section{Discusión}

El modelo inicialmente hipotetizado de la SMAN (M0), constituido por los 5 factores o constructos y los 20 ítems, mostró diferencias estadísticamente significativas con la matriz de datos observada $(p=0,006, \leq 0,05)$, y se encontraron covarianzas de error mal especificadas entre los ítems FRD4 y FRP1, FRPNF3 y FRP4, lo que llevó a hacer una reespecificación del modelo.

Para la reespecificación, se tuvieron en cuenta los parámetros planteados por Shek y Yu [52], donde se describen dos alternativas: establecer rutas entre las covarianzas de los ítems pertenecientes a un mismo constructo sugeridos como modificables o eliminar aquellos ítems cuya covarianza de error estimada presente una carga cruzada con ítems de otros factores.

Durante la reespecificación, se evidenció que, en el factor relacionado con la discapacidad, el ítem FRD4, "Asisto a mi proceso de rehabilitación, así se presenten otros trastornos de salud adicionales a los que padezco", midió no solo cómo el paciente percibe su compromiso en el proceso de rehabilitación, sino también el ítem FRP1 del factor relacionado con el paciente: "Mantengo motivado en mi proceso de rehabilitación”. Este hallazgo podría deberse a una estrecha relación de los factores personales, donde la motivación se relaciona 
con la percepción de cumplimiento, compromiso y la autoeficacia en los procesos de rehabilitación [53-56].

También se evidencio que el ítem FRP4, "Tengo esperanza frente a mi proceso de rehabilitación”, no solo midió el constructo de factores relacionados con el paciente, sino también el constructo de procesos de neurorrehabilitación funcional correspondiente al ítem FRPNF3, "Asisto a las sesiones programadas de rehabilitación, así aún no vea cambios en mi proceso de rehabilitación". Esto puede deberse a la capacidad percibida de los sujetos de generar intenciones hacia las metas deseadas, además de una sobrevaloración de los eventos positivos, en los que se incluye la futura rehabilitación de la condición de salud y que se define como sesgo de optimismo. También estaría relacionado con la posibilidad de mostrar la mejor imagen de sí mismo en el contexto del estudio y podría interpretarse como un sesgo de respuesta de deseabilidad social [57-59].

El análisis teórico realizado a los ítems demostró la necesidad de eliminar aquellos que generaban redundancia dentro del instrumento. La eliminación no afecto la consistencia de los constructos, dado que el modelo definitivo conservó el número de factores del modelo inicial y un mínimo de 3 ítems por factor, según lo planteado por Lloret et al. [38]. La calificación de la adherencia con el instrumento SMAN, posterior a la eliminación de los 2 ítems, se ajustó teniendo en cuenta un rango entre 18 y 90 puntos, donde el cálculo de la adherencia final se mantiene con respecto al instrumento inicialmente publicado [37].

El ajuste del modelo o el último modelo propuesto (M2), fue evaluado por las medidas de ajuste absoluto, medidas de ajuste incremental y medidas de ajuste de la parsimonia, sugeridas por Byrne [42], Wheaton et al. [44] y Hooper et al. [45]. En las medidas de ajuste absoluto, el chi cuadrado relativo $\left(\chi^{2} / g l\right)$ se distinguió por un valor inferior a 2 ; índices como el GFI y AGFI mostraron valores cercanos a 1, y el SRMR y el RMSEA se ubicaron con valores inferiores al criterio de 0,05 , exhibiendo entonces un buen ajuste del modelo. Para las medidas NFI, CFI, TLI e IFI se obtuvieron valores por encima del criterio de 0,90 , demostrando así que el modelo tiene una buena bondad de ajuste incremental. Las medidas de ajuste a la parsimonia (PGFI, PNFI) demostraron valores por encima del criterio de 0,50 y los índices AIC y ECVI presentaron disminución en los valores en los dos modelos M1 y M2. Lo anterior demostró tener un punto óptimo sobre la complejidad del modelo y una mejor bondad de ajuste de parsimonia.

La fiabilidad y la validez convergente del modelo M2 de la SMAN, establecidas a través del AC, IFC y AVE, cumplieron con los criterios de referencia planteados, lo que demuestra que los ítems miden satisfactoriamente el constructo evaluado, teniendo en cuenta la influencia de los otros constructos sobre el medido. La validez dis- criminante calculada demostró correlación significativa, positiva y alta entre todos los constructos, determinando la diferencia y la relación entre los conceptos que conforman la SMAN.

La presente investigación genera un aporte valioso a los procesos de rehabilitación, al determinar la fiabilidad y la validez de un instrumento para evaluar de manera confiable la adherencia a procesos de neurorrehabilitación funcional. Si bien el acceso a la población que asiste a dichos procesos no es fácil, dada la baja prevalencia de las enfermedades neurológicas y la carencia de registros sistemáticos de seguimiento - siendo esta una de las limitantes que presentó este estudio_-, esta investigación obtuvo una muestra suficiente para establecer la validez de los constructos que configuran la SMAN y constituye un aporte al personal asistencial, debido a que sus resultados pueden ser usados para predecir tempranamente los motivos de incumplimiento y no adherencia de las personas adultas a sus tratamientos.

Dadas las consideraciones anteriores, la adherencia a los procesos terapéuticos resultan de la adecuada relación y la dinámica establecida por los actores de las dimensiones; considerar las necesidades y prioridades del usuario es quizá la clave para la permanencia y el logro de objetivos en el abordaje. Este instrumento es un soporte para ser usasdo en la toma de decisiones frente a la trazabilidad de objetivos hacia la rehabilitación funcional.

Se puede concluir que la evidencia teórica previamente descrita, los hallazgos empíricos del presente estudio y el marco estadístico de la validez y la fiabilidad a través de un AFC sugieren que la SMAN, constituida por 5 factores o constructos, y 18 ítems, posee propiedades psicométricas destacables y confiables para la medición de la adherencia en poblaciones con déficit funcional asociado a condición neurológica.

\section{Agradecimientos}

A las personas que aceptaron voluntariamente a participar en el estudio, a ellas, nuestro más magno y fraterno agradecimiento. Adicionalmente, le agradecemos a la Institución Educativa Escuela Nacional del Deporte por apoyar esta iniciativa.

\section{Declaración de fuente de financiación}

Se declara que esta investigación fue financiada con recursos de la Institución Educativa Escuela Nacional del Deporte.

\section{Declaración de conflictos de intereses}

Los autores manifiestan no tener conflicto de intereses. 


\section{Declaración de responsabilidad}

Se declara que los puntos de vista expresados en este artículo son responsabilidad de los autores y no de la institución donde trabajan.

\section{Declaración de contribución por autores}

Los autores declaramos cumplir con los criterios del International Committee of Medical Journal Editors (ICMJE) para ser autores del presente escrito. Carlos A. Quiroz participó en el procesamiento estadístico de los datos, en la escritura del método y los resultados. Mauricio Hernández realizó el procesamiento estadístico de los datos y elaboró el modelo de ecuaciones estructurales; también participó en la escritura del método y de los resultados. Zulamy Agudelo y Carlos Medina participaron en la escritura y la redacción del resumen, la introducción y la discusión. Así mismo, cada autor está en la capacidad y la disposición de responder por el contenido del manuscrito y su proceso de elaboración.

\section{Referencias}

1. World Health Organization, editor. Neurological disorders: Public health challenges. Geneva: World Health Organization; 2006.

2. Jin J, Sklar GE, et al. Factors affecting therapeutic compliance: A review from the patient's perspective. Ther Clin Risk Manag. 2008;4:269-86. Dor: https://doi.org/10.2147/tcrm.s1458

3. Organización Mundial de la Salud. Adherencia a los tratamientos a largo plazo. Pruebas para la acción. Ginebra: Organización Panamericana de la Salud [internet]; 2004 [citado 2020 abr. 14]. Disponible en: https://www.paho.org/spanish/ad/dpc/nc/adherencia-largo-plazo.pdf

4. Coetzee N, Andrewes D, Khan F, et al. Predicting compliance with treatment following stroke: A new model of adherence following rehabilitation. Brain Impair: Cambridge University Pres. 2008;9(2):122-39. DoI: https://doi.org/10.1375/brim.9.2.122

5. Bassett SF. The assessment of patient adherence to physiotherapy rehabilitation. NZ Journal of Physiotherapy. 2003 [citado 2020 abr. 27]; 31(2):60-66. Disponible en: https://www.researchgate. net/publication/284411604_The_assessment_of_patient_adherence_to_physiotherapy_rehabilitation

6. Duncan PW, Horner RD, Reker DM, et al. Adherence to postacute rehabilitation guidelines is associated with functional recovery in stroke. Stroke. 2002;33(1):167-78. DoI: https://doi.org/10.1161/ hs 0102.101014

7. Mapipa HV. Identifying contributing factors for poor adherence to rehabilitative services in patients with neurological conditions [dissertation]. [Pretoria]: University of Pretoria [internet]; 2014 [citado 2020 may. 14]. Disponible en: https://repository.up.ac.za/ handle/2263/46138

8. Duncan TE, McAuley E. Social support and efficacy cognitions in exercise adherence: A latent growth curve analysis. J Behav Med. 1993;16:199-218. Dor: https://doi.org/10.1007/BF00844893

9. Frier A, Barnett F, Devine S. The relationship between social determinants of health, and rehabilitation of neurological conditions: a systematic literature review. Disabil Rehabil. 2017(39)10:941-8. DOI: https://doi.org/10.3109/09638288.2016.1172672

10. Zinn S. Patient adherence in rehabilitation. H. Bosworth, E. Oddone, M. Weinberger, editors. Patient treatment adherence. Concepts, interventions, and measurement. Londres: Taylor \& Francis; 2008. pp. 195-236 DoI: https://doi.org/10.4324/9781410615626

11. Jurkiewicz MT, Marzolini S, Oh P. Adherence to a home-based exercise program for individuals after stroke. Top Stroke Rehabil. 2011;18(3):277-84. DOI: https://doi.org/10.1310/tsr1803-277

12. Pishkhani MK, Dalvandi A, et al. Adherence to a rehabilitation regimen in stroke patients: A concept analysis. Iran J Nurs Midwifery Res. 2020;25:139-45. DoI: https://doi.org/10.4103/ijnmr. IJNMR $170 \quad 18$

13. Munce SEP, Graham ID, Salbach NM, et al. Perspectives of health care professionals on the facilitators and barriers to the implementation of a stroke rehabilitation guidelines cluster randomized controlled trial. BMC. Health Serv Res. 2017;17:440. DoI: https://doi. org/10.1186/s12913-017-2389-7

14. Koudriavtseva T, Onesti E, Pestalozza IF, et al. The importance of physician-patient relationship for improvement of adherence to long-term therapy: Data of survey in a cohort of multiple sclerosis patients with mild and moderate disability. Neurol Sci. 2012;33:575-84. DoI: https://doi.org/10.1007/s10072-011-0776-0

15. Khoshbakht Pishkhani M, Dalvandi A, Ebadi A, Hosseini M. Factors affecting adherence to rehabilitation in Iranian stroke patients: A qualitative study. J Vasc Nurs 2019;37(4):264-71. DoI: https:// doi.org/10.1016/j.jvn.2019.07.001

16. Wentink MM, Meesters J, Berger MAM, et al. Adherence of stroke patients with an online brain training program: The role of health professionals' support. Top Stroke Rehabil. 2018;25(5):359-65. DOI: https://doi.org/10.1080/10749357.2018.1459362

17. Turner AP, Knowles LM. Behavioral interventions in multiple sclerosis. Fed Pract. [internet]. 2020 [citado 2020 abr. 28]; 37(Suppl. 1):S31-S35. Disponible en: https://www.ncbi.nlm.nih. gov/pmc/articles/PMC7182244/

18. Allen NE, Song J, Paul SS, et al. Predictors of adherence to a falls prevention exercise program for people with Parkinson's disease. Mov Disord Clin Pract. 2015;2(4):395-401. Dor: https://doi. org/10.1002/mdc3.12208

19. Colombo R, Pisano F, Mazzone A, et al. Design strategies to improve patient motivation during robot-aided rehabilitation. J NeuroEngineering Rehabil. 2007;4:3. DoI: https://doi. org/10.1186/1743-0003-4-3

20. Suttanon P, Hill KD, Said CM, et al. Factors influencing commencement and adherence to a home-based balance exercise program for reducing risk of falls: Perceptions of people with Alzheimer's disease and their caregivers. Int Psychogeriatr. 2012;24(7):117282. Dor: https://doi.org/10.1017/S1041610211002729

21. Yao M, Chen J, Jing J, Sheng H, Tan X, Jin J. Defining the rehabilitation adherence curve and adherence phases of stroke patients: An observational study. Patient Prefer Adherence. 2017;11:143541. DoI: https://doi.org/10.2147/PPA.S139854

22. Letendre SL, Ellis RJ, et al. Neurologic complications of HIV disease and their treatment. Top HIV Med. 2010;18(2):45-55.

23. Essery R, Geraghty AWA, et al. Predictors of adherence to homebased physical therapies: A systematic review. Disabil Rehabil. 2017;39(6):519-34. DoI: https://doi.org/10.3109/09638288.2016. 1153160

24. Glass SP, Matin N, Williams B, et al. Neuropsychiatric factors linked to adherence and short-term outcome in a U.S. functional neurological disorders clinic: A retrospective cohort study. J 
Neuropsychiatry Clin Neurosci. 2017;30:152-9. Dor: https://doi. org/10.1176/appi.neuropsych.17060117

25. Tiedemann A, Sherrington C, Dean CM, et al. Predictors of adherence to a structured exercise program and physical activity participation in community dwellers after stroke. Stroke Res Treat. 2012;2012. DoI: https://doi.org/10.1155/2012/136525

26. Amofa Sr PA, DeFeis B, De Wit L, et al. Functional ability is associated with higher adherence to behavioral interventions in mild cognitive impairment. Clin Neuropsychol. 2019;34(5):937-955. DOI: https://doi.org/10.1080/13854046.2019.1672792

27. Silva MA, Calvo D, Brennan EM, et al. Incidence and predictors of adherence to sleep apnea treatment in rehabilitation inpatients with acquired brain injury. Sleep Med 2020;69:159-67. DOI: https://doi.org/10.1016/j.sleep.2020.01.016

28. Grindley EJ, Zizzi SJ, Nasypany AM. Use of protection motivation theory, affect, and barriers to understand and predict adherence to outpatient rehabilitation. Phys Ther. 2008;88(12):1529-40. DOI: https://doi.org/10.2522/ptj.20070076

29. Grindley EJ, Zizzi SJ. Using a multidimensional approach to predict motivation and adherence to rehabilitation in older adults. Top Geriatr Rehabil. 2005;21(3):182-93. Dor: https://doi. org/10.1097/00013614-200507000-00004

30. Kernan WN. Keeping Faith With Patients After Stroke or Transient Ischemic Attack. JAMA Neurol. 2018;75(4):404-5. DOI: https://doi.org/10.1001/jamaneurol.2017.4027

31. Clay DL, Hopps JA. Treatment adherence in rehabilitation: The role of treatment accommodation. Rehabil Psychol. 2003;48(3):215-9. DoI: https://doi.org/10.1037/0090-5550.48.3.215

32. Iuga AO, McGuire MJ. Adherence and health care costs. Risk Manag Healthc Policy. 2014;7:35-44. DoI: https://doi.org/10.2147/ RMHP.S19801

33. Martín Alfonso L. Repercusiones para la salud pública de la adherencia terapéutica deficiente. Rev Cuba Salud Pública [internet]. 2006 [citado 2020 may. 13]; 32(3). Disponible en: http://scielo.sld.cu/scielo.php?script=sci_arttext\&pid $=$ S0864-34662006000300013

34. Silva GE, Galeano E, Correa JO. Adherencia al tratamiento. Implicaciones de la no-adherencia. Acta Méd Colomb. [internet] 2005 [citado 2020 abr. 14]; 30(4):268-73. Disponible en: https:// www.redalyc.org/pdf/1631/163113820004.pdf

35. Frost R, Levati S, McClurg D, et al. What adherence measures should be used in trials of home-based rehabilitation interventions? A systematic review of the validity, reliability, and acceptability of measures. Arch Phys Med Rehabil. 2017;98(6):1241-56. e45. DoI: https://doi.org/10.1016/j.apmr.2016.08.482

36. Quiroz CA. Instrumento para evaluar adherencia de personas adultas a procesos de neurorehabilitación funcional basado en las dimensiones propuestas de la Organización Mundial de la Salud. Rev Fac Nac de Salud Pública [internet]. 2014 [citado 2020 abr. 14]; 32(1):52-61. Disponible en: https://revistas.udea.edu.co/index.php/fnsp/article/view/13378

37. Quiroz CA, Perez JE. Reliability instrument to assess adherence to adult long term functional neurorehabilitation process (SMAN 1.0). Physiotherapy. 2015;101(Supl. 1):e1245-6. DoI: https://doi. org/10.1016/j.physio.2015.03.1147

38. Lloret-Segura S, Ferreres-Traver A, et al. El análisis factorial exploratorio de los ítems: una guía práctica, revisada y actualizada. An Psicol. 2014;30(3):1151-69. Dor: https://doi.org/10.6018/analesps.30.3.199361
39. Batista-Foguet JM, Coenders G, Alonso J. Análisis factorial confirmatorio. Su utilidad en la validación de cuestionarios relacionados con la salud. Med Clín. 2004;122(Supl. 1):21-27. Dor: https:// doi.org/10.1157/13057542

40. Ryu E. Effects of skewness and kurtosis on normal-theory based maximum likelihood test statistic in multilevel structural equation modeling. Behav Res Methods. 2011;43:1066-74. Dor: https://doi. org/10.3758/s13428-011-0115-7

41. Rodríguez-Ayán MN, Ruiz-Díaz MA. Atenuación de la asimetría $\mathrm{y}$ de la curtosis de las puntuaciones observadas mediante transformaciones de variables: incidencia sobre la estructura factorial. Psicológica [internet]. 2008 [citado 2020 abr. 14]; 29(2): 205-27. Disponible en: https://www.redalyc.org/pdf/169/16929206.pdf

42. Byrne BM. Structural equation modeling with AMos, basic concepts, applications and programming. $3 .^{a}$ ed. Nueva York: Routledge [internet]; 2016 [citado 2020 may. 14]. Disponible en: https://crispindia.org/wp-content/uploads/2016/11/Structuralequation-modeling-with-AMOS.pdf

43. Cole JC, Motivala SJ, Khanna D, et al. Validation of single-factor structure and scoring protocol for the health assessment questionnaire-disability index. Arthritis Care Res. 2005;53(4):536-42. DoI: https://doi.org/10.1002/art.21325

44. Wheaton B, Muthén B, et al. Assessing reliability and stability in panel models. Sociol Methodol. 1977;8:84-136. Dor: https://doi. org/10.2307/270754

45. Hooper D, Coughlan J, Mullen MR. Structural equation mode1ling: Guidelines for determining model fit. Electron J Bus Res Methods [internet] 2008 [citado 2020 abr. 14]; 6(1):53-60. Disponible en: https://arrow.tudublin.ie/buschmanart/2/

46. Hu L, Bentler PM. Cutoff criteria for fit indexes in covariance structure analysis: Conventional criteria versus new alternatives. Struct Equ Model Multidiscip J. 1999;6(1):1-55. Dor: https://doi. org/10.1080/10705519909540118

47. Henseler J, Ringle CM, Sarstedt M. A new criterion for assessing discriminant validity in variance-based structural equation modeling. J Acad Mark Sci. 2015;43:115-35. Dor: https://doi. org/10.1007/s11747-014-0403-8

48. Oviedo HC, Campo-Arias A. Aproximación al uso del coeficiente alfa de Cronbach. Rev Colomb Psiquiatr. [internet]. 2005 [citado 2020 abr. 14]; 34(4):572-80. Disponible en: https://www.redalyc. org/pdf/806/80634409.pdf

49. Dunn TJ, Baguley T, Brunsden V. From alpha to omega: A practical solution to the pervasive problem of internal consistency estimation. Br J Psychol. 2014;105(3):399-412. Dor: https://doi. org/10.1111/bjop. 12046

50. Fornell C, Larcker DF. Evaluating structural equation models with unobservable variables and measurement error. J Mark Res. 1981;18(1):39-50. DoI: https://doi. org/10.1177/002224378101800104

51. Prieto G, Delgado AR. Fiabilidad y validez. Papeles Psicólogo [internet]. 2010 [citado 2020 abr. 14]; 31(1):67-74. Disponible en: http://www.papelesdelpsicologo.es/pdf/1797.pdf

52. Shek DTL, Yu L. Confirmatory factor analysis using Amos: A demonstration. Int J Disabil Hum Dev. 2014;13(2):191-204. DoI: https://doi.org/10.1515/ijdhd-2014-0305

53. Rossini Y, Estrada O, et al. Factores relacionados con adherencia a un programa de rehabilitación. RFS Rev Fac Salud. 2010;2(1):3950. DoI: https://doi.org/10.25054/rfs.v2i1.28 
54. Maclean N, Pound $\mathrm{P}$, et al. The concept of patient motivation. Stroke. 2002;33(2):444-8. DoI: https://doi.org/10.1161/ hs0202.102367

55. Bandura A. The explanatory and predictive scope of self-efficacy theory. J Soc Clin Psychol. 1986;4(3):359-73. DoI: https://doi org/10.1521/jscp.1986.4.3.359

56. Morris JH, Oliver T, Kroll T, Joice S, Williams B. Physical activity participation in community dwelling stroke survivors: Synergy and dissonance between motivation and capability. A qualitative study. Physiotherapy. 2017;103(3):311-21. Dor: https://doi. org/10.1016/j.physio.2016.05.001
57. Levy T, Laver K, Killington M, et al. A systematic review of measures of adherence to physical exercise recommendations in people with stroke. Clin Rehabil. 2019;33(3):535-45. Dor: https:// doi.org/10.1177/0269215518811903

58. Sharot T. The optimism bias. Curr Biol. 2011;21(23):R941-5. DOI: https://doi.org/10.1016/j.cub.2011.10.030

59. Van de Mortel TF. Faking it: Social desirability response bias in self-report research. Australian J Advanced Nursing [internet]. 2005 [citado 2020 abr. 14]; 25(4): 40-48. Disponible en: https:// www.ajan.com.au/archive/Vol25/Vol_25-4_vandeMortel.pdf 
Anexo. Instrumento de evaluación de la adherencia a los procesos de neurorrehabilitación: SMAN v2.0

Número de entrevista:
Nombre y apellidos:
Fecha de entrevista: $\frac{1}{\text { Mes/Día/Año }}$

Diagnóstico:

Fecha del diagnóstico u ocurrencia del trastorno:

Fecha de inicio del proceso de rehabilitación:

\begin{tabular}{|c|c|c|c|c|c|c|}
\hline Factor & Ítem & Nunca & Casi nunca & A veces & Casi siempre & Siempre \\
\hline \multirow{4}{*}{$\begin{array}{l}\text { O } \\
. \frac{0}{E} \\
.0 \\
\overline{0} \\
0 \\
\mathbb{d} \\
. \frac{0}{0} \\
0 \\
0\end{array}$} & Cuento con recursos para mi proceso de rehabilitación & 1 & 2 & 3 & 4 & 5 \\
\hline & $\begin{array}{l}\text { Mis redes sociales (del barrio, de la iglesia, del trabajo, } \\
\text { del colegio), favorecen mis procesos de rehabilitación }\end{array}$ & 1 & 2 & 3 & 4 & 5 \\
\hline & $\begin{array}{l}\text { Sigo mi proceso de rehabilitación, a pesar de las } \\
\text { creencias que tengan otras personas sobre mi } \\
\text { enfermedad }\end{array}$ & 1 & 2 & 3 & 4 & 5 \\
\hline & $\begin{array}{l}\text { Cuento con el apoyo de mi familia para mi proceso de } \\
\text { neurorrehabilitación }\end{array}$ & 1 & 2 & 3 & 4 & 5 \\
\hline \multirow{4}{*}{ 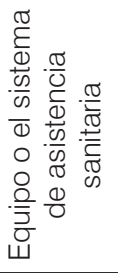 } & Mi servicio de salud facilita mi proceso de rehabilitación & 1 & 2 & 3 & 4 & 5 \\
\hline & $\begin{array}{l}\text { La preparación y el conocimiento de los profesionales } \\
\text { que me asisten favorece mi proceso de rehabilitación }\end{array}$ & 1 & 2 & 3 & 4 & 5 \\
\hline & $\begin{array}{l}\text { El personal que me asiste hace seguimiento a mi } \\
\text { condición de salud y mi proceso de rehabilitación }\end{array}$ & 1 & 2 & 3 & 4 & 5 \\
\hline & $\begin{array}{l}\text { El personal que me asiste demuestra interés por mi } \\
\text { proceso de rehabilitación }\end{array}$ & 1 & 2 & 3 & 4 & 5 \\
\hline \multirow{4}{*}{ 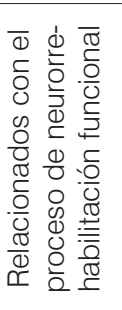 } & $\begin{array}{l}\text { Recibo las sesiones necesarias para mi proceso de } \\
\text { rehabilitación }\end{array}$ & 1 & 2 & 3 & 4 & 5 \\
\hline & Asisto a las sesiones programadas de rehabilitación & 1 & 2 & 3 & 4 & 5 \\
\hline & $\begin{array}{l}\text { Asisto a las sesiones programadas de rehabilitación, } \\
\text { así aun no vea cambios en mi proceso de } \\
\text { rehabilitación }\end{array}$ & 1 & 2 & 3 & 4 & 5 \\
\hline & $\begin{array}{l}\text { Asisto a las sesiones programadas de rehabilitación, a } \\
\text { pesar de los posibles efectos secundarios }\end{array}$ & 1 & 2 & 3 & 4 & 5 \\
\hline \multirow{3}{*}{ 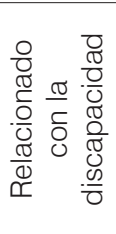 } & $\begin{array}{l}\text { Asisto a mi proceso de rehabilitación, a pesar de mi } \\
\text { condición funcional }\end{array}$ & 1 & 2 & 3 & 4 & 5 \\
\hline & $\begin{array}{l}\text { Asisto a mi proceso de rehabilitación, independiente de } \\
\text { la progresión de mi enfermedad }\end{array}$ & 1 & 2 & 3 & 4 & 5 \\
\hline & $\begin{array}{l}\text { Asisto a mi proceso de rehabilitación, así mi } \\
\text { enfermedad sea grave }\end{array}$ & 1 & 2 & 3 & 4 & 5 \\
\hline \multirow{3}{*}{ 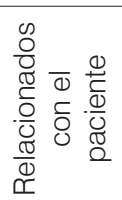 } & Mantengo motivado en mi proceso de rehabilitación & 1 & 2 & 3 & 4 & 5 \\
\hline & $\begin{array}{l}\text { Reconozco la necesidad de mi proceso de } \\
\text { rehabilitación }\end{array}$ & 1 & 2 & 3 & 4 & 5 \\
\hline & $\begin{array}{l}\text { Entiendo las instrucciones dadas respecto a mi } \\
\text { proceso de rehabilitación }\end{array}$ & 1 & 2 & 3 & 4 & 5 \\
\hline
\end{tabular}

PUNTAJE FINAL: (Escala 18 - 90 puntos)

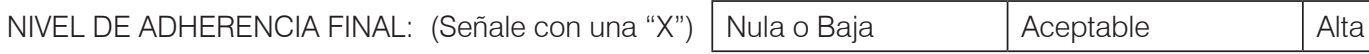

Para calcular la Adherencia final utilice la siguiente fórmula: $\quad x=\frac{\left(\sum \text { ítems }-18\right) * 100}{72}$

Donde, Adherencia final es igual a la sumatoria de todos los ítems, menos 18 puntos, por cien, dividido setenta y dos. Para determinar el Nivel de Adherencia final se sugiere que utilice la siguiente escala:

\begin{tabular}{|l|c|}
\hline Nula o baja & $0-49 \%$ \\
\hline Aceptable & $50-74 \%$ \\
\hline Alta & $75-100 \%$ \\
\hline
\end{tabular}

